\title{
Infección Urinaria en Pacientes con Lesión Medular
}

\section{Urinary Infection in Patients with Spinal Cord Lesion}

\author{
Irma A. Ospina-Galeano ${ }^{1}$ Albert Borau Duran ${ }^{2}$ \\ ${ }^{1}$ Servicio de Neurourología y Urología Funcional, Hospital Pablo \\ Tobón Uribe, Medellín, Colombia \\ 2 Servicio de Urología, Instituto Guttmann, Badalona, España \\ Address for correspondence Irma A. Ospina-Galeano, MD, Urologist, \\ Servicio de Neurourología y Urología Funcional, Hospital Pablo Tobón \\ Uribe, CALLE 78 B NO. 69-240, Medellín, Colombia \\ (e-mail: irmaospinagalenao@gmail.com).
}

Urol Colomb 2018;27:8-13.

\section{Resumen}

Palabras Clave

- infección urinaria

- lesión medular

- bacteriuria asintomática

- cateterismo intermitente

- disfunción vesical

- vejiga neurogénica

\section{Abstract}

La micción es un proceso complejo, que requiere la coordinación entre el sistema nervioso central y periférico. La alteración en ése, aumenta el riesgo para que se produzcan infecciones complicadas y a largo plazo, daño renal. La alteración en el vaciado de la vejiga, obliga a que muchos de esos pacientes, realicen cateterismos intermitentes o sonda vesical permanente, aumentando el riesgo de infecciones polimicrobianas o por gérmenes multiresistentes. Algunos factores implicados en el desarrollo de las infecciones de esos pacientes, son el residuo postmiccional elevado, estasis urinario, litiasis vesical, uso de catéteres, además de las alteraciones en el sistema inmune y las capas de recubrimiento en la mucosa vesical. El diagnóstico de infección se realiza al encontrar: piuria y bacteriuria, según método de vaciado vesical, y un síntoma general que sugiera infección. Conclusión: Las infecciones urinarias en pacientes con lesión medular, deben ser tratadas de acuerdo a sensibilidades de la zona, siempre con la toma previa de un urocultivo y con un diagnóstico adecuado de infección urinaria, teniendo en cuenta las diferentes maniobras de evacuación de la vejiga. No están recomendados los tratamientos cortos ni el tratamiento de las bacteriurias asintomáticas. Una de las formas de prevenir las infecciones, está en evitar situaciones de riesgo como el estasis vesical, las presiones intravesicales elevadas y los vaciamientos incompletos. En la actualidad existen múltiples medicamentos para prevenir las infecciones urinarias, pero faltan estudios con evidencia de más peso y en pacientes con lesión medular, para que puedan ser recomendados.

Urination is a complex process, requiring coordination between the central and peripheral nervous system. The alteration in this, increases the risk for complicated infections and long-term kidney damage. The alteration in the emptying of the bladder, causes many of these patients to perform intermittent catheterization or permanent bladder catheterization, increasing the risk of polymicrobial infections or multiresistant received

February 28, 2017

accepted

August 4, 2017
DOI https://doi.org/ $10.1055 / \mathrm{s}-0038-1637014$ ISSN 0120-789X. eISSN 2027-0119.
Copyright (c) 2018, Sociedad Colombiana License terms de Urología. Publicado por Thieme Revinter Publicações Ltda., Rio de Janeiro, Brazil. Todos los derechos reservados. 


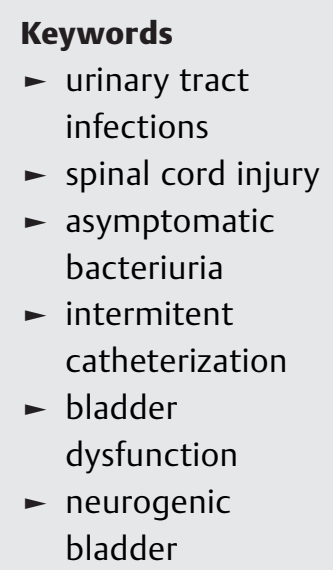

germs. Some factors involved in the development of infections of these patients are high postvoiding, urinary stasis, vesical lithiasis, use of catheters, as well as alterations in the immune system and the layers of lining in the bladder mucosa. The diagnosis of infection is made on finding: pyuria and bacteriuria, according to the method of bladder emptying, and a general symptom that suggests infection. Conclusion: Urinary tract infections in patients with spinal cord injury should be treated according to the sensitivity of the area, always with prior urine collection and an adequate diagnosis of urinary tract infection, taking into account the different maneuvers of bladder evacuation. Short treatments and treatment of asymptomatic bacteriuria are not recommended. One way to prevent infections is to avoid risky situations such as bladder stasis, elevated intravesical pressures and incomplete emptying. At the moment there are multiple drugs to prevent urinary tract infections, but there are no studies with evidence of heaviest weight and in patients with spinal cord injury, so that they can be recommended.

\section{Introducción}

La micción es un proceso complejo, que requiere la coordinación entre el sistema nervioso central y periférico.

La alteración en ese proceso, aumenta el riesgo para que se produzcan infecciones complicadas y a largo plazo, daño renal. Aproximadamente, del $10 \%$ al $15 \%$ de los pacientes con vejiga neurogénica, mueren por sepsis de origen urinario. ${ }^{1,2}$

Esa alteración en el vaciado de la vejiga, obliga a que muchos de esos pacientes, realicen cateterismos intermitentes o a que usen sonda vesical permanente, hechos que aumentan el riesgo de infección del tracto urinario (ITU).

En nuestro medio, tenemos una creciente población de pacientes con enfermedades neurológicas y lesiones medulares, que requieren ingresos hospitalarios y por lo tanto, están expuestos a ser colonizados o tener infecciones por gérmenes multiresistentes.

Existen varios factores implicados en el desarrollo de las infecciones urinarias en los pacientes con lesión medular. Se consideran el residuo postmiccional elevado, estasis urinario, litiasis vesical, uso de catéteres, además de las alteraciones en el sistema inmune.

El objetivo de este artículo es hacer una revisión donde podamos identificar factores de riesgo, y repasar conceptos relacionados con el diagnóstico, tratamiento y prevención de las infecciones urinarias en pacientes con lesión medular.

\section{Epidemiología}

La infección urinaria es la infección más común en los pacientes con enfermedades neurológicas. En el primer año posterior a una lesión medular, el 31\% de los pacientes son diagnosticados de ITU, y el 21\% requieren hospitalización. ${ }^{1}$

A nivel nacional no se tiene bien identificada la incidencia de la lesión medular. Carvajal, en un estudio realizado en un hospital de tercer nivel de Medellín, describe que la población principalmente afectada, está entre la segunda y tercera década de la vida. Halla una relación hombre:mujer de 9:1, y que por 1000 pacientes ingresados en UCI, 12 presentan una lesión medular. $^{3}$
Los pacientes con lesión medular tienen alrededor de 2,5 infecciones sintomáticas al año. Encontrándose que en adultos sanos, los principales microorganismos aislados corresponden a gram negativos como Eschericia coli y Klebsiella y en los pacientes con vejiga neurogénica, aumenta la tasa de infección por otros microorganismos como Pseudomona (15\%), Acinetobacter (15\%), Enterococcus (6\%) o infecciones polimicrobianas (26\%). Esos hechos nos indican que el tratamiento para los pacientes con vejiga neurogénica, debe ser orientado de forma especial. ${ }^{4-7}$

\section{Patogénesis y Factores de Riesgo}

\section{Patogénesis}

Se identificaron deficiencias en factores inmunes como el CD14 M y D88 cuando existe lesión medular, lo cual implica una alteración primaria en el sistema de reconocimiento de patógenos a la hora de controlar la infección. También en la respuesta pro y antiinflamatoria de la infección aguda. ${ }^{8}$

Se ha observado alteración en la secreción de inmunoglobulina $\mathrm{A}$, la cual es la principal mediadora de la inmunidad humoral en las mucosas.

Hay otros factores, como la alteración de la flora nativa de la vagina y del periné, que actúan como barrera protectora, para que otros uropatógenos no colonicen la orina. Desde 1970 se ha investigado el papel de la capa de glucosaminoglicano GAG, observando que sus defectos dejan expuestas la células epiteliales a los componentes de la orina siendo el primer paso para la invasión bacteriana y el desencadenamiento de la ITU y las enfermedades inflamatorias crónicas que comprometen la vejiga.

Esa capa, puede estar además afectada por la sobredistensión vesical, las altas presiones vesicales, el uso de catéteres y la inflamación crónica. ${ }^{9-11}$

\section{Factores de Riesgo}

La disfunción vesical al generar altas presiones, produce isquemia en los propios tejidos, retrasando la respuesta inmune a los patógenos. Además, la isquemia hace que disminuya el flujo sanguíneo hasta 1,2 veces en los tejidos 
vesicales, favoreciendo la expresión de factores de crecimiento que aumentan la fibrosis y posteriormente disminuyen la acomodación vesical. ${ }^{12,13}$

Se ha visto además, que el vaciamiento incompleto contribuye en esa patología. En un estudio se observó que después de introducir bacterias en la vejiga de cerdos sanos, el 99\% de ellas eran eliminadas durante la micción. Otro estudio encontró una relación directa entre el volumen residual y el riesgo de ITU, encontrando que con volúmenes por encima de $300 \mathrm{ml}$, el riesgo de ITU puede aumentar hasta 4 o 5 veces, al favorecer la permanencia en vejiga de bacterias uropatógenas. ${ }^{14}$

El reflujo vesicoureteral también aumenta el riesgo, pero no existe un consenso sobre cuál es el valor que debemos considerar como significativo. ${ }^{4}$

Dentro de los diferentes métodos de vaciamiento, existe una preferencia por el cateterismo intermitente sobre los que incluyen sonda permanente, sonda suprapúbica, urocolector externo, micción refleja y maniobras de Credé o Valsalva. Cuando se realiza el cateterismo, las bacterias pueden entrar directamente en la vejiga con el catéter, generando daño permanente en la mucosa vesical, además de daño en la capa de GAG. Al realizarse el vaciamiento vesical por el catéter y no por los mecanismos de vaciado normales, la orina no puede limpiar la uretra. La biopelícula que se forma alrededor de los catéteres permanentes, hace que los microorganismos desarrollen resistencias a los mecanismos de defensa del huésped y también a los antimicrobianos. ${ }^{15,16}$

Se observó que en pacientes con lesión medular, el número de episodios de ITU por cada 100 pacientes/día, según el sistema de vaciado, fue de 2,72 si usaban sonda permanente, 0,41 cateterismo intermitente, 0,36 colector externo, 0,34 catéter suprapúbico y 0,06 en casos con vaciamiento espontáneo.

Aunque con el colector externo se presente casi la misma tasa de infección urinaria que con el cateterismo intermitente, probablemente no se realiza un vaciado completo de la vejiga como ocurre en el cateterismo, por lo que esa indicación debe hacerse individualizada, basándose en la evaluación clínica de cada paciente. 2,4,17,18 $^{2}$

Se ha encontrado que el nivel de la lesión puede ser otro factor de riesgo para ITU, algunos autores han informado que los pacientes tetrapléjicos tienen 2,5 veces más riesgo de infección que los parapléjicos y esos últimos más que los que tienen lesión en el cono medular. Se ha visto además, que los pacientes con lesiones completas tienen dos veces más riesgo de ITU que los de lesiones incompletas, probablemente porque tienen un menor grado de independencia y mayor riesgo a requerir cateterización. ${ }^{19}$

Un estudio comparó la incidencia de ITU, en pacientes con lesión medular alta que usaban cateterismo intermitente o sonda suprapúbica, hallando una tasa del $26 \%$ y $12 \%$ respectivamente, teniendo los usuarios de sonda suprapúbica, una incidencia mayor de litiasis vesical. ${ }^{17,20,21}$

\section{Diagnóstico}

En la actualidad, no existe un consenso para definir la infección urinaria en los pacientes con vejiga neurogénica, ya que existen síntomas inespecíficos y múltiples definiciones. ${ }^{5}$
La definición de bacteriuria, según un consenso del Instituto Nacional de Discapacidad e Investigación de Rehabilitación (NDIRR/USA), es: $>10^{2}$ UFC en personas con cateterismo intermitente, $>10^{4}$ UFC para los que llevan urocolector y cualquier aislamiento en pacientes con catéter permanente 0 punción suprapúbica. Para realizar el diagnóstico de ITU debe existir además piuria, definido como 5 o más leucocitos por campo y cualquier signo o síntoma de infección. ${ }^{22-24}$

Los síntomas que generalmente se presentan en esa población, son tales como: disreflexia autonómica, incremento de la espasticidad, nuevos episodios de incontinencia urinaria o su empeoramiento, orina fétida, con sedimento o dolor abdominal.

La ausencia de piuria, sugiere fuertemente otra causa de los síntomas por su alta sensibilidad (82,2\%). La orina fétida y con sedimento, tiene una alta especificidad (79\% y 83\% respectivamente), y la más alta precisión la tienen la disreflexia y la fiebre.

Se evaluó qué tan precisos podían ser los síntomas a la hora de detectar una ITU, se identificó que los pacientes tenían mejor predicción de no tener infección que que si la tuvieran, reafirmando la dificultad para el diagnóstico en ese tipo de pacientes. ${ }^{25,26}$

La infección podría definirse entonces, en los casos que se encuentre: piuria y bacteriuria, según método de vaciado vesical, y un síntoma general que sugiera infección. ${ }^{18}$

El seguimiento que se hace a los pacientes con lesión medular, debe incluir periódicamente, estudios para evaluar además de su función renal, anormalidades del tracto urinario, dinámica miccional y la presencia de infecciones urinarias. $2,18,27,28$

\section{Tratamiento}

La colonización bacteriana en los pacientes con lesión medular, es la norma por el uso de catéteres y su sola presencia, no es indicación de tratamiento ya que él aumenta el riesgo de infecciones recurrentes y el desarrollo de resistencias. ${ }^{5,29,30}$ Siempre antes de iniciar cualquier tratamiento, se debe realizar un urocultivo. La elección del antibiótico empírico, estará basado en los patógenos y sensibilidades específicas de la zona y posteriormente, se harán ajustes con los resultados obtenidos.

En los Estados Unidos, se ha observado que solo el 18\% de los pacientes con lesión medular, tenían ITU sintomática por E. Coli, comparado con un $75 \%-95 \%$ de la población general, ya que los pacientes con lesión medular, suelen tener ITU por múltiples microorganismos. ${ }^{25}$ Por tanto, los antibióticos de amplio espectro, deben ser considerados para tratar a esos pacientes, al presentar infecciones de bacterias con cepas multiresistentes.

En caso de que el área tenga alta prevalencia de resistencia a las quinolonas, ellas deben ser usadas con precaución. ${ }^{31}$

Se debe evitar el tratamiento de las bacteriurias asintomáticas en pacientes con lesión medular, excepto en inmunosuprimidos, durante el embarazo o si son sometidos a procedimientos urológicos. Los ciclos continuos de antibióticos dan como resultado altas tasas de resistencia antibiótica, y las 
profilaxis antibióticas tienen eficacia limitada, estando más asociadas a la resistencia. ${ }^{29}$

La Sociedad Americana de Enfermedades Infecciosas, recomendó el cambio temprano de catéteres entre los 7 y los 14 días del tratamiento en curso, con obtención de cultivos específicos. Se encontró en un estudio, que los cursos cortos de 5 días, con cambio de catéter en pacientes con sonda permanente, se asociaban más a recaídas infecciosas. La recomendación que se realiza basado en varios estudios a la fecha, es de realizar tratamientos largos en pacientes con lesión medular. La duración del tratamiento debe estar entre 7-14 días, dependiendo de la respuesta al tratamiento instaurado. Las terapias duales que incluyen aminoglicósidos se deben reservar para pacientes con sepsis grave. ${ }^{22,32}$

\section{Prevención}

\section{Cateterismo Intermitente}

En el paciente con lesión medular, debe realizarse en promedio, un cateterismo cada 4- 6 horas. Controlar siempre los volúmenes de riesgo para ITU, que estarían por encima de $500 \mathrm{~mL}$ y los volúmenes que no desencadenen presiones intravesicales elevadas, que puedan ser de riesgo para daño a largo plazo del tracto urinario superior.

Esas presiones pueden ser definidas mediante el estudio urodinámico que se le realiza al paciente como evaluación inicial clasificatoria, cuando ya ha pasado el shock medular. ${ }^{4,5,10,27}$

No se ha podido demostrar que sea mejor en cuanto a la aparición de ITU asociada al catéter, si se practica una técnica estéril en comparación a la práctica de una técnica limpia. Sin embargo, en un consenso americano, se recomienda una técnica estéril cuando el paciente presenta ITU recurrentes sintomáticas. ${ }^{18,22,33,34}$

\section{Tipo de Catéter}

En los estudios realizados hasta el momento, se ha visto que el uso de catéteres hidrofílicos, retrasa la aparición de la primera ITU en pacientes con lesión medular aguda. En cambio, a largo plazo, no se han encontrado diferencias en cuanto a la incidencia de ITU, si el paciente usa catéter hidrofílico o catéter no cubierto. También se ha encontrado disminución en el microtrauma uretral medido por hematuria en los catéteres hidrofílicos, pero no se logró demostrar que los catéteres cubiertos tuvieran menos fricción que los no cubiertos. Los cateteres reutilizables con una buena técnica de limpieza pueden ser apropiados para el cateterismo intermitente, sin que se aumente el riesgo de ITU. ${ }^{35-37}$

En cuanto al uso de catéteres recubiertos con plata o con antibióticos, no se han encontrado ventajas en la aparición de ITU asociada al catéter, por lo tanto, aún no existe evidencia para preferir su uso. ${ }^{38,39}$

\section{Catéteres Permanentes}

Se ha visto que los pacientes que usan catéter permanente tienen menos riesgo de hidronefrosis y mejor acomodación, si se le adicionan anticolinérgicos. ${ }^{5}$
Con respecto a los catéteres suprapúbicos, se han realizado varias revisiones del tema, encontrando que pueden ser una alternativa para algunos pacientes que deseen más independencia, que estén más cómodos con su imagen corporal, que no toleren los cateterismos o que hayan tenido traumas uretrales o hipospadias. Podremos tener una mejor capacidad y funcionalidad si se adicionan anticolinérgicos y realizan clampajes periódicos para mantener volúmenes funcionales, además de realizar cistoscopias de rutina para identificar a tiempo complicaciones que se pueden presentar con ese tipo de derivaciones. Sin embargo el catéter permanente por vía uretral, también puede ser una alternativa válida para algunos pacientes. ${ }^{17,21,40-42}$

En cuanto al material del catéter, no existe uno que sea biocompatible o libre de complicaciones, pero si se ha visto que existe más riesgo de aumentar la respuesta inflamatoria y generar cistitis glandular cuando se usan los catéteres de látex. ${ }^{22}$

\section{Arándano y D- Manosa}

Se conocen los efectos in vitro de las proantocianidinas del arándano rojo en la prevención de la adherencia por fibra $\mathrm{P}$ uropatogénica de Escherichia coli, y la relación dosis respuesta en la disminución de la virulencia de la bacteria.

Según una revisión de Cochrane 2012, los estudios realizados hasta el momento, no han logrado demostrar el beneficio del uso rutinario del arándano en la prevención de ITU en pacientes con vejiga neurogénica. $22,43,44$

$\mathrm{Al}$ analizar mujeres con ITU recurrentes, se encontró que una toma diaria de D-manosa, reduce la incidencia de ITU en un $45 \%$ a los 6 meses, comparando con la profilaxis con nitrofurantoína, pero no se han realizado estudios similares en pacientes con vejiga neurogénica.

Un posible mecanismo para explicar la falta de eficacia del arándano y la D- manosa, es que en la población neurogénica se encuentran varios uropatógenos generando la infección, y el Arándano actúa principalmente sobre E. coli y la D manosa sobre Pseudomona. ${ }^{9}$

\section{Interferencia Bacteriana}

Se han probado clínicamente 2 agentes microbianos que pueden generar interferencia bacteriana para prevenir la ITU, son ellos: E. coli 83972 la cual no expresa la P fimbria y HU2117, la cual fue creada desde una cepa salvaje de E. coli 83792 con una mutación en el gen que expresa la P fimbria. Hemos comprobado con datos in Vitro, que esas bacterias tienen la habilidad de prevenir la formación de biopelículas por lo uropatogenos en los catéteres urinarios.

En un ensayo clínico realizado por Darouiche y col., donde se inoculaba cepa de E. coli HU 2117, que es menos virulenta en pacientes con lesión medular, se encontró que existía menos riesgo de ITU sintomática. Ahora, dirigimos nuestra investigación en el sentido de encontrar vías efectivas y más fáciles para colonizar la vejiga. ${ }^{45,46}$

\section{Probióticos Lactobacillus}

Desde que correlacionaron las altas concentraciones de lactobacilos y las bajas tasas de ITU, se promovió el uso de probióticos que contienen lactobacilos y restauran la flora 
vaginal nativa, como un tratamiento y profilaxis en las infecciones urogenitales bacterianas. Los estudios de interferencia con probióticos de lactobacilos, se han realizado principalmente en mujeres, pues en los hombres, los resultados no han sido exitosos.

También se evaluó el efecto en mujeres postmenopáusicas, donde no se encontró inferioridad, comparado con la profilaxis antibiótica. $^{47}$

Recientemente se realizó un ensayo clínico que comparó la profilaxis para ITU, con diferentes lactobacilos y placebos en pacientes neurogénicos, encontrando que ésa es una terapia efectiva para prevenir la ITU y la colonización por gérmenes multiresistentes. ${ }^{48}$

Instilación vesical: Se ha visto en mujeres con vejigas no neurogénicas, una disminución del 86,6\% en los episodios de ITU con instilaciones vesicales de ácido hialurónico semanal o mensual comparado con placebo, pero esos estudios no han sido extrapolados a pacientes con lesión medular. ${ }^{11,49}$

Profilaxis antibiótica: La profilaxis antibiótica es controversial ya que se debe valorar la eficacia a largo plazo, y el desarrollo emergente de las resistencias antibióticas. Por ahora no se recomienda esa práctica, por el contrario, sí que se recomiendan los tratamientos completos. $5,22,28,30,50$

\section{Conclusiones}

Las infecciones urinarias en los pacientes con lesión medular, deben ser tratadas de acuerdo a sensibilidades de la zona, siempre con la toma previa de un urocultivo y con un diagnóstico adecuado de infección urinaria, teniendo en cuenta las diferentes maniobras para evacuar la vejiga del paciente. No están recomendados los tratamientos cortos ni el tratamiento de las bacteriurias asintomáticas.

Una de las formas de prevenir las infecciones está en evitar situaciones de riesgo como el estasis vesical, las presiones intravesicales elevadas y los vaciamientos incompletos. En la actualidad existen múltiples medicamentos para prevenir las infecciones urinarias, pero faltan estudios con evidencia de más peso y en pacientes con lesión medular, para que puedan ser recomendados.

Conflictos de Intereses

Los autores declaramos que no existen conflictos de intereses.

\section{Referencias}

1 McKibben MJ, Seed P, Ross SS, Borawski KM. Urinary Tract Infection and Neurogenic Bladder. Urol Clin North Am 2015;42 (04):527-536

2 Carvajal C, Pacheco C, Gómez-Rojo C, Calderón J, Cadavid C, Jaimes F. Clinical and demographic characteristics of patients with spinal cord injury Six years experience. Acta Med Colomb 2015;40(01):45-50

3 Esclarín De Ruz A, García Leoni E, Herruzo Cabrera R; DE RUZ AE. Epidemiology and risk factors for urinary tract infection in patients with spinal cord injury. J Urol 2000;164(04):1285-1289

4 Goetz LL, Klausner AP. Strategies for prevention of urinary tract infections in neurogenic bladder dysfunction. Phys Med Rehabil Clin N Am 2014;25(03):605-618, viii
5 García Leoni ME, Esclarín De Ruz A. Management of urinary tract infection in patients with spinal cord injuries. Clin Microbiol Infect 2003;9(08):780-785

6 Mukai S, Shigemura K, Nomi M, et al. Retrospective study for risk factors for febrile UTI in spinal cord injury patients with routine concomitant intermittent catheterization in outpatient settings. Spinal Cord 2016;54(01):69-72

7 Chaudhry R, Madden-Fuentes RJ, Ortiz TK, et al. Inflammatory response to Escherichia coli urinary tract infection in the neurogenic bladder of the spinal cord injured host. J Urol 2014; 191(05):1454-1461

8 Jahromi MS, Mure A, Gomez CS. UTIs in patients with neurogenic bladder. Curr Urol Rep 2014;15(09):433

9 Vasudeva P, Madersbacher H. Factors implicated in pathogenesis of urinary tract infections in neurogenic bladders: some revered, few forgotten, others ignored. Neurourol Urodyn 2014;33(01):95-100

10 Cicione A, Cantiello F, Ucciero G, et al. Intravesical treatment with highly-concentrated hyaluronic acid and chondroitin sulphate in patients with recurrent urinary tract infections: Results from a multicentre survey. Can Urol Assoc J 2014;8(9-10):E721-E727

11 Kershen RT, Azadzoi KM, Siroky MB. Blood flow, pressure and compliance in the male human bladder.JUrol 2002;168(01):121-125

12 Nicolle LE. Urinary tract infections in special populations: diabetes, renal transplant, HIV infection, and spinal cord injury. Infect Dis Clin North Am 2014;28(01):91-104

13 Kim B-R, Lim JH, Lee SA, et al. The Relation between Postvoid Residual and Occurrence of Urinary Tract Infection after Stroke in Rehabilitation Unit. Ann Rehabil Med 2012;36(02):248-253

14 Berry RE, Klumpp DJ, Schaeffer AJ. Urothelial cultures support intracellular bacterial community formation by uropathogenic Escherichia coli. Infect Immun 2009;77(07):2762-2772

15 Leuck A-M, Wright D, Ellingson L, Kraemer L, Kuskowski MA, Johnson JR. Complications of Foley catheters-is infection the greatest risk? J Urol 2012;187(05):1662-1666

16 Nomura S, Ishido T, Teranishi J, Makiyama K. Long-term analysis of suprapubic cystostomy drainage in patients with neurogenic bladder. Urol Int 2000;65(04):185-189

17 Consortium for Spinal Cord Medicine. Paralyzed Veterans of America. Bladder management for adults with spinal cord injury: a clinical practice guideline for health-care providers. Washington, DC: Consortium for Spinal Cord Medicine; 2006

18 Çetinel B, Önal B, Can G, Talat Z, Erhan B, Gündüz B. Risk factors predicting upper urinary tract deterioration in patients with spinal cord injury: A retrospective study: Risk Factors for Upper Urinary Tract Deterioration. Neurourol Urodyn 2017;36(03):653-658

19 Siroky MB. Pathogenesis of bacteriuria and infection in the spinal cord injured patient. Am J Med 2002;113(1, Suppl 1A)67S-79S

20 Mitsui T, Minami K, Furuno T, Morita H, Koyanagi T. Is suprapubic cystostomy an optimal urinary management in high quadriplegics? A comparative study of suprapubic cystostomy and clean intermittent catheterization. Eur Urol 2000;38(04):434-438

21 Hunter KF, Bharmal A, Moore KN. Long-term bladder drainage: Suprapubic catheter versus other methods: a scoping review. Neurourol Urodyn 2013;32(07):944-951

22 Hooton TM, Bradley SF, Cardenas DD, et al; Infectious Diseases Society of America. Diagnosis, prevention, and treatment of catheter-associated urinary tract infection in adults: 2009 International Clinical Practice Guidelines from the Infectious Diseases Society of America. Clin Infect Dis 2010;50(05):625-663

23 James D. The prevention and management of Urinary Tract Infection among people with spinal cord injuries. Disabil Rehabil Res 1992;1:5-36

24 Ferroni M, Taylor AK. Asymptomatic Bacteriuria in Noncatheterized Adults. Urol Clin North Am 2015;42(04):537-545

25 Massa LM, Hoffman JM, Cardenas DD. Validity, accuracy, and predictive value of urinary tract infection signs and symptoms in individuals with spinal cord injury on intermittent catheterization. J Spinal Cord Med 2009;32(05):568-573 
26 Linsenmeyer TA, Oakley A. Accuracy of individuals with spinal cord injury at predicting urinary tract infections based on their symptoms. J Spinal Cord Med 2003;26(04):352-357

27 Cameron AP, Rodriguez GM, Schomer KG. Systematic review of urological followup after spinal cord injury. J Urol 2012;187(02): 391-397

28 Posible complications of urinary incontinence in people with neurological disease. National Institute for Health and Care Excellence. 2015: 2-7

29 Boyle DP, Zembower TR. Epidemiology and Management of Emerging Drug-Resistant Gram-Negative Bacteria: ExtendedSpectrum $\beta$-Lactamases and Beyond. Urol Clin North Am 2015; 42(04):493-505

30 Nicolle LE. Urinary tract infections in patients with spinal injuries. Curr Infect Dis Rep 2014;16(01):390-399

31 Yoon SB, Lee BS, Lee KD, Hwang SI, Lee HJ, Han ZA. Comparison of bacterial strains and antibiotic susceptibilities in urinary isolates of spinal cord injury patients from the community and hospital. Spinal Cord 2014;52(04):298-301

32 Dinh A, Toumi A, Blanc C, et al. Management of febrile urinary tract infection among spinal cord injured patients. BMC Infect Dis 2016;16(01):156-162

33 Hudson E, Murahata RI. The 'no-touch' method of intermittent urinary catheter insertion: can it reduce the risk of bacteria entering the bladder? Spinal Cord 2005;43(10):611-614

34 Fasugba O, Koerner J, Mitchell BG, Gardner A. Systematic review and meta-analysis of the effectiveness of antiseptic agents for meatal cleaning in the prevention of catheter-associated urinary tract infections. J Hosp Infect 2017;95(03):233-242

35 Cardenas DD, Moore KN, Dannels-McClure A, et al. Intermittent catheterization with a hydrophilic-coated catheter delays urinary tract infections in acute spinal cord injury: a prospective, randomized, multicenter trial. PM R 2011;3(05):408-417

36 Bermingham SL, Hodgkinson S, Wright S, Hayter E, Spinks J, Pellowe C. Intermittent self catheterisation with hydrophilic, gel reservoir, and non-coated catheters: a systematic review and cost effectiveness analysis. BMJ 2013;346(15):e8639

37 Stensballe J, Looms D, Nielsen PN, Tvede M. Hydrophilic-coated catheters for intermittent catheterisation reduce urethral micro trauma: a prospective, randomised, participant-blinded, crossover study of three different types of catheters. Eur Urol 2005;48(06): 978-983
38 Pickard R, Lam T, MacLennan G, et al. Antimicrobial catheters for reduction of symptomatic urinary tract infection in adults requiring short-term catheterisation in hospital: a multicentre randomised controlled trial. Lancet 2012;380(9857):1927-1935

39 Salameh A, Al Mohajer M, Daroucihe RO. Prevention of urinary tract infections in patients with spinal cord injury. CMAJ 2015; 187(11):807-811

40 Feifer A, Corcos J. Contemporary role of suprapubic cystostomy in treatment of neuropathic bladder dysfunction in spinal cord injured patients. Neurourol Urodyn 2008;27(06):475-479

41 Sorokin I, De E. Options for independent bladder management in patients with spinal cord injury and hand function prohibiting intermittent catheterization. Neurourol Urodyn 2015;34(02):167-176

42 Katsumi HK, Kalisvaart JF, Ronningen LD, Hovey RM. Urethral versus suprapubic catheter: choosing the best bladder management for male spinal cord injury patients with indwelling catheters. Spinal Cord 2010;48(04):325-329

43 Freire GdeC, Williams G, Craig JC. Cranberries for preventing urinary tract infections. Sao Paulo Med J 2013;131(05):363

44 Berger RE. Cranberries for preventing urinary tract infections. J Urol 2005;173(06):1988

45 Darouiche RO, Green BG, Donovan WH, et al. Multicenter randomized controlled trial of bacterial interference for prevention of urinary tract infection in patients with neurogenic bladder. Urology 2011;78(02):341-346

46 Darouiche RO, Hull RA. Bacterial interference for prevention of urinary tract infection. Clin Infect Dis 2012;55(10):1400-1407

47 Beerepoot MAJ, ter Riet G, Nys S, et al. Lactobacilli vs antibiotics to prevent urinary tract infections: a randomized, double-blind, noninferiority trial in postmenopausal women. Arch Intern Med 2012;172(09):704-712

48 Lee BB, Toh S-L, Ryan S, et al. Probiotics [LGG-BB12 or RC14-GR1] versus placebo as prophylaxis for urinary tract infection in persons with spinal cord injury [ProSCIUTTU]: a study protocol for a randomised controlled trial. BMC Urol 2016;16:18-26

49 Damiano R, Quarto G, Bava I, et al. Prevention of recurrent urinary tract infections by intravesical administration of hyaluronic acid and chondroitin sulphate: a placebo-controlled randomised trial. Eur Urol 2011;59(04):645-651

50 Blok B, Pannek J, Diaz DC, del Popolo G, Groen J, Gross T. Neurourology. 2015; Available from: http://uroweb.org/wp-content/ uploads/21-Neuro-Urology_LR2.pdf 